\title{
Practical Error Analysis of Cross-Ratio-Based Planar Localization
}

\author{
Jen-Hui Chuang, Jau-Hong Kao, Horng-Horng Lin, and Yu-Ting Chiu \\ Deptartment of Computer Science, National Chiao-Tung University, \\ No. 1001, Ta-Hseuh Rd., Hsinchu, Taiwan \\ \{jchuang, hhlin\}@cs.nctu.edu.tw, gis88804@cis.nctu.edu.tw, \\ cloude.cs94g@nctu.edu.tw
}

\begin{abstract}
Recently, more and more computer vision researchers are paying attention to error analysis so as to fulfill various accuracy requirements arising from different applications. As a geometric invariant under projective transformations, cross-ratio is the basis of many recognition and reconstruction algorithms which are based on projective geometry. We propose an efficient way of analyzing localization error for computer vision systems which use cross-ratios in planar localization. By studying the inaccuracy associated with cross-ratio-based computations, we inspect the possibility of using linear transformation to approximate localization error due to 2-D noises of image extraction for reference points. Based on such a computationally efficient analysis, a practical way of choosing point features in an image so as to establish the probabilistically most accurate planar location system using crossratios is developed.
\end{abstract}

Keywords: cross-ratio, error analysis, error ellipse, robot localization.

\section{Introduction}

One of the main purposes of computer vision is to develop a reliable system that can carry out its tasks, e.g., reconstruction of scene structures, with satisfactory efficiency and precision in a realistic environment. There are basically two classes of methods to reconstruct 3-D structures from 2-D images. The first class involves strategies relying on camera calibration to establish reconstruction matrices while the second class consists of approaches based on projective geometry associated with reference points given as prior knowledge.

As a geometric invariant under projective transformations, cross-ratio is the basis of many recognition and reconstruction algorithms which are based on projective geometry [1][2]. For example, cross-ratios calculated from vertices of polygons are used in [3-7] to recognize planar features in a 3-D environment. In addition to recognition, given prior knowledge about a scene, object structure can also be reconstructed using cross-ratio. For example, an approach that transforms relative affine structure defined in [8] into equivalent cross-ratio measurement is used to determine relative 3-D face structure from facial images in an identity recognition system [9]. Such a projective invariant can also be utilized to match trajectories across video streams and applied to image retrieval problems [10][11]. For autonomous 
navigation of vehicles, cross-ratio is often used to identify artificial landmarks or beacons placed in the environment [12-16].

As indicated in [17][18], the quality of scene reconstruction and structure inference strongly depends on the quality of the image data. In addition to other possible measurement uncertainties, 2-D coordinates of feature points in an image plane will always have quantization errors due to limited image resolution. Hence, values of projective coordinates, i.e., pairs of cross-ratios with respect to some given reference points, will also be noisy. Some studies of cross-ratio are proposed to assess its use in invariant-based recognition systems [17-21]. These studies mainly focus on robust estimations of the cross-ratio but not the final localization or reconstruction results for autonomous navigation applications.

In this paper, we propose a novel way of analyzing localization error for systems which use cross-ratio for planar localization. Through a 1 st-order approximation of the derived one dimensional error function, we first inspect the linear nature of localization error due to small inaccuracy in image data. Similar properties of the localization error due to two dimensional noises are then investigated. In particular, an approximation of a nominal boundary of error ellipse can be determined efficiently for one of image points being affected by radially symmetric errors of a fixed magnitude. Based on such a computationally efficient error analysis, one may obtain the picture of resultant regions of localization error in advance, and select proper reference image points accordingly.

The rest of the paper is organized as follows. In Section 2, we introduce a general invariant-based method for 3-D reconstruction of locations of planar features using cross-ratio. Subsequently, the localization error due to associated cross-ratio computation using noisy 2-D image data is formulated in Section 3. We determine how the error propagates, including the direction and magnitude range, through linear approximation of such a cross-ratio-based formulation. In Section 4, synthesized noise is added to real data in the experiments for the verification of the related theoretical investigations. Finally, a summary is given in Section 5.

\section{Projective Invariant-Based 3-D Reconstruction}

Before the description of a typical framework for 3-D reconstruction of a scene point from four reference points using projective invariant cross-ratio, we first review some mathematics involved in its computation. Let $O, A, B, C$ and $D$ be five coplanar points

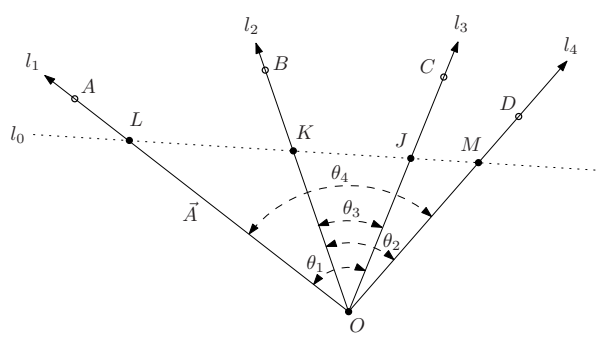

Fig. 1. Cross-ratio of five coplanar points 
in a general configuration (with no three of them being collinear), as shown in Fig. 1. One form of cross-ratio can be computed as ${ }^{1}$

$$
[A, B, C, D]_{O} \triangleq C R_{O} \triangleq \frac{\sin \theta_{1} \sin \theta_{2}}{\sin \theta_{3} \sin \theta_{4}}=\frac{|\vec{A} \times \vec{C}||\vec{B} \times \vec{D}|}{|\vec{B} \times \vec{C}||\vec{A} \times \vec{D}|}
$$

with $O$ being the origin of pencils $\overrightarrow{O A}, \overrightarrow{O B}, \overrightarrow{O C}, \overrightarrow{O D}$. Let $\vec{A}=\left(A_{x}, A_{y}\right)$ stands for vector $\overleftrightarrow{O A}$, and so on. We can rewrite (1) as

$$
[A, B, C, D]_{O}=\left(\left|\begin{array}{cc}
A_{x} & C_{x} \\
A_{y} & C_{y}
\end{array}\right|\left|\begin{array}{cc}
B_{x} & D_{x} \\
B_{y} & D_{y}
\end{array}\right|\right) /\left(\left|\begin{array}{cc}
B_{x} & C_{x} \\
B_{y} & C_{y}
\end{array}\right|\left|\begin{array}{cc}
A_{x} & D_{x} \\
A_{y} & D_{y}
\end{array}\right|\right) \triangleq\left(K_{A C} K_{B D}\right) /\left(K_{B C} K_{A D}\right)=Q_{1} / Q_{2} .
$$

Thus, a cross-ratio can be obtained without computing the sinusoidals in (1). Given five scene points $O, A, B, C, D$ located on a 3 -D plane $\pi_{0}$, with no three of them being collinear, they are projected on image plane $\pi_{1}$ as $o, a, b, c$ and $d$, respectively ${ }^{2,3}$. The invariant property of cross-ratio, assures that if the five feature points can be identified in the image plane accurately, the cross-ratio $[a, b, c, d]_{o}$ will be identical to the cross-ratio $[A, B, C, D]_{O}$.

An immediate application of the projective invariant cross-ratio is to determine ray directions. For example, if the origin and three of the rest four points are known in Fig. 1, the vector passing through the fourth point from the origin can be determined easily if $[A, B, C, D]_{O}$ is given. For example, let $D=(X, Y)$, or $\overrightarrow{O D}$, is to be determined. From (1), we have

$$
C R_{O}=K_{A C}\left|\begin{array}{cc}
B_{x} & X-O_{x} \\
B_{y} & Y-O_{y}
\end{array}\right| / K_{B C}\left|\begin{array}{cc}
A_{x} & X-O_{x} \\
A_{y} & Y-O_{y}
\end{array}\right| \triangleq \frac{Q_{1}}{Q_{2}}
$$

which can be rewritten as

$$
\left(Q_{1} K_{B C} A_{y}-Q_{2} K_{A C} B_{y}\right) X+\left(Q_{2} K_{A C} B_{x}-Q_{1} K_{B C} A_{x}\right) Y=Q_{2} K_{A C}\left|\begin{array}{ll}
B_{x} & O_{x} \\
B_{y} & O_{y}
\end{array}\right|-Q_{1} K_{B C}\left|\begin{array}{ll}
A_{x} & O_{x} \\
A_{y} & O_{y}
\end{array}\right| .
$$

This is in fact the equation of $l_{4}(\overleftrightarrow{O D})$.

Furthermore, if $O, A, B, C$ are known, so as $[A, B, C, D]_{O}$ and $[B, C, D, O]_{A}$, we can obtain point $D$ by intersecting $\overrightarrow{O D}$ and $\overrightarrow{A D}$. Accordingly, a localization system can be developed based on the invariant cross-ratio, assuming perfect image acquisition and feature extraction. However, measurement uncertainty and system noise, such as quantization errors of 2-D coordinates of feature points in an image plane due to limited image resolution, usually occur in practice. These uncertainties will propagate

\footnotetext{
${ }^{1}$ Note that a total of 24 different cross-ratios $k_{i}, 1 \leq i \leq 24$, can be defined for a scene point and Eq. (1) corresponds to $k_{1}$ defined in [21].

${ }^{2}$ In the rest of the paper, we denote image points with lowercase letters and scene points with uppercase letters.

${ }^{3}$ In a robot navigation environment, $\pi_{0}$ can be the ground plane and the five points can be landmarks or beacons placed in the environment, or the robot itself.
} 
through the computation process, resulting in erroneous localizations or reconstructions. In the next section, we will investigate how measurement errors may propagate in a reconstruction process based on cross-ratio.

\section{Error Analysis of Cross-Ratio-Based Localization}

A general configuration of coplanar points for cross-ratio-based localization is shown in Fig. 2. Assume $P_{1}, P_{2}, P_{3}, P_{4}$ are known planar points in 3-D space with $P_{1}$ and $P_{4}$, as well as $p_{1}$ and $p_{4}$, being origins for two corresponding cross-ratios. The position of a scene point $R$ (or the location of a robot), which corresponds to image point $r$, can be determined with the procedure described in Section 2. For simplicity, let $\overrightarrow{p_{1} r}=\left(d_{x}, d_{y}\right)$ and assume the location of $p_{4}$ has noise $\Delta x$ along $x$-direction and is extracted as $\hat{p}_{4}$, we have

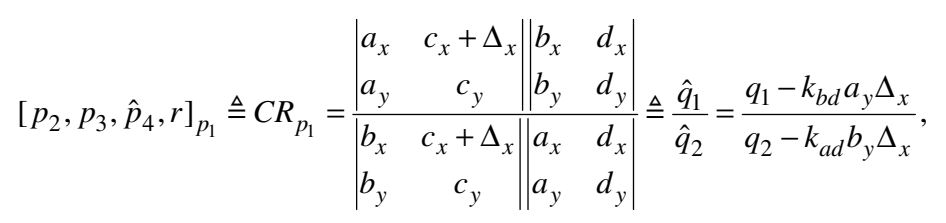

where $k_{b d}=\left|\begin{array}{ll}b_{x} & d_{x} \\ b_{y} & d_{y}\end{array}\right|$ and $k_{a d}=\left|\begin{array}{ll}a_{x} & d_{x} \\ a_{y} & d_{y}\end{array}\right|$. Substituted $Q_{1}$ and $Q_{2}$ in (3) by $\hat{q}_{1}$ and $\hat{q}_{2}$,

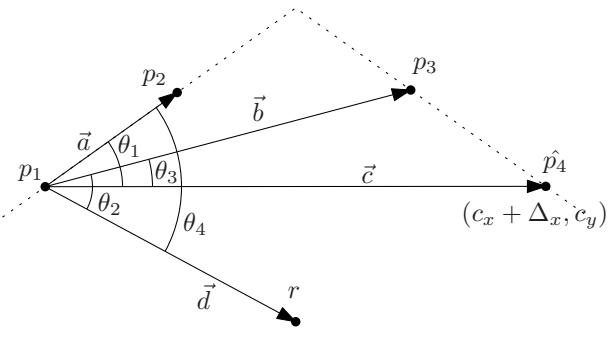

Fig. 2. A general configuration of coplanar points where $p_{1}$ is the origin of four pencils

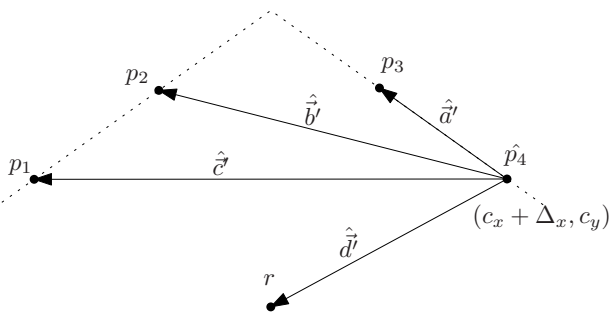

Fig. 3. $\hat{p}_{4}$ is used as the origin to compute cross-ratio $C R_{\hat{p}_{4}}$ 
we have

$$
\left(\hat{q}_{1} K_{B C} A_{y}-\hat{q}_{2} K_{A C} B_{y}\right) X+\left(\hat{q}_{2} K_{A C} B_{x}-\hat{q}_{1} K_{B C} A_{x}\right) Y=\hat{q}_{2} K_{A C}\left|\begin{array}{cc}
B_{x} & P_{1 x} \\
B_{y} & P_{1 y}
\end{array}\right|-\hat{q}_{1} K_{B C}\left|\begin{array}{cc}
A_{x} & P_{1 x} \\
A_{y} & P_{1 y}
\end{array}\right|
$$

which yields line equation of $\overleftrightarrow{P_{1} R}$

$$
\begin{aligned}
& {\left[\left(q_{1}-k_{b d} a_{y} \Delta_{x}\right) K_{B C} A_{y}-\left(q_{2}-k_{a d} b_{y} \Delta_{x}\right) K_{A C} B_{y}\right] X+} \\
& {\left[\left(q_{2}-k_{a d} b_{y} \Delta_{x}\right) K_{A C} B_{x}-\left(q_{1}-k_{b d} a_{y} \Delta_{x}\right) K_{B C} A_{x}\right] Y} \\
& =\left(q_{2}-k_{a d} b_{y} \Delta_{x}\right) K_{A C}\left|\begin{array}{ll}
B_{x} & P_{1 x} \\
B_{y} & P_{1 y}
\end{array}\right|-\left(q_{1}-k_{b d} a_{y} \Delta_{x}\right) K_{B C}\left|\begin{array}{ll}
A_{x} & P_{1 x} \\
A_{y} & P_{1 y}
\end{array}\right| .
\end{aligned}
$$

On the other hand, with $\hat{p}_{4}$ being the origin, as shown in Fig. $3, C R_{\hat{p}_{4}}$ can be computed as

$$
\frac{\left|\begin{array}{cc}
a_{x}^{\prime}-\Delta_{x} & c_{x}^{\prime}-\Delta_{x} \\
a_{y}^{\prime} & c_{y}^{\prime}
\end{array}\right|\left|\begin{array}{cc}
b_{x}^{\prime}-\Delta_{x} & d_{x}^{\prime}-\Delta_{x} \\
b_{y}^{\prime} & d_{y}^{\prime}
\end{array}\right|}{\left|\begin{array}{ccc}
b_{x}^{\prime}-\Delta_{x} & c_{x}^{\prime}-\Delta_{x} \\
b_{y}^{\prime} & c_{y}^{\prime}
\end{array}\right| \begin{array}{cc}
a_{x}^{\prime}-\Delta_{x} & d_{x}^{\prime}-\Delta_{x} \\
a_{y}^{\prime} & d_{y}^{\prime}
\end{array} \mid} \triangleq \frac{\hat{q}_{1}^{\prime}}{\hat{q}_{2}^{\prime}}=\frac{q_{1}^{\prime}+u_{1} k_{a c}^{\prime} \Delta_{x}+u_{2} k_{b d}^{\prime} \Delta_{x}+u_{1} u_{2} \Delta_{x}^{2}}{q_{2}^{\prime}+u_{3} k_{b c}^{\prime} \Delta_{x}+u_{4} k_{a d}^{\prime} \Delta_{x}+u_{3} u_{4} \Delta_{x}^{2}},
$$

where $u_{1}=b_{y}^{\prime}-d_{y}^{\prime}, u_{2}=a_{y}^{\prime}-c_{y}^{\prime}, u_{3}=a_{y}^{\prime}-d_{y}^{\prime}, u_{4}=b_{y}^{\prime}-c_{y}^{\prime}$. Similarly, from (3) and (6), we can obtain line equation of $\overrightarrow{\hat{P}_{4} R}$ as

$$
\begin{aligned}
& \left(\hat{q}_{1}^{\prime} K_{B C}^{\prime} A_{y}^{\prime}-\hat{q}_{2}^{\prime} K_{A C}^{\prime} B_{y}^{\prime}\right) X+\left(\hat{q}_{2}^{\prime} K_{A C}^{\prime} B_{x}^{\prime}-\hat{q}_{1}^{\prime} K_{B C}^{\prime} A_{x}^{\prime}\right) Y \\
& =\hat{q}_{2}^{\prime} K_{A C}^{\prime}\left|\begin{array}{ll}
B_{x}^{\prime} & P_{4 x} \\
B_{y^{\prime}} & P_{4 y}
\end{array}\right|-\hat{q}_{1}^{\prime} K_{B C}^{\prime}\left|\begin{array}{cc}
A_{x}^{\prime} & P_{4 x} \\
A_{y}^{\prime} & P_{4 y}
\end{array}\right|
\end{aligned}
$$

where $\left(P_{4 x}, P_{4 y}\right)$ is coordinate of the scene point $P_{4}$. It is easy to see that (5) and (7) are of the form

$$
\left\{\begin{array}{c}
\alpha_{1}^{\prime} X+\beta_{1}^{\prime} Y=\gamma_{1}^{\prime} \\
\alpha_{2}^{\prime} X+\beta_{2}^{\prime} Y=\gamma_{2}^{\prime}
\end{array}\right.
$$

Therefore, by solving the above equations, the robot position can be obtained as

$$
R \triangleq\left(R_{x}, R_{y}\right)=\left(\left|\begin{array}{ll}
\gamma_{1}^{\prime} & \beta_{1}^{\prime} \\
\gamma_{2}^{\prime} & \beta_{2}^{\prime}
\end{array}\right| /\left|\begin{array}{cc}
\alpha_{1}^{\prime} & \beta_{1}^{\prime} \\
\alpha_{2}^{\prime} & \beta_{2}^{\prime}
\end{array}\right|,\left|\begin{array}{ll}
\alpha_{1}^{\prime} & \gamma_{1}^{\prime} \\
\alpha_{2}^{\prime} & \gamma_{2}^{\prime}
\end{array}\right| /\left|\begin{array}{ll}
\alpha_{1}^{\prime} & \beta_{1}^{\prime} \\
\alpha_{2}^{\prime} & \beta_{2}^{\prime}
\end{array}\right|\right)
$$

To simplify (8), by skipping high order terms of $\Delta x$, we have

$$
\begin{gathered}
\left|\begin{array}{ll}
\gamma_{1}^{\prime} & \beta_{1}^{\prime} \\
\gamma_{2} & \beta_{2}^{\prime}
\end{array}\right| \approx\left|\begin{array}{ll}
\gamma_{1} & \beta_{1} \\
\gamma_{2} & \beta_{2}
\end{array}\right|+\Delta_{x}\left[M_{1}\left(\left|\begin{array}{ll}
P_{4 x} A_{x} & A_{x}^{\prime} \\
P_{1 x} A_{y} & A_{y}^{\prime}
\end{array}\right|+A_{x} A_{x}^{\prime}\left(P_{1 y}-P_{4 y}\right)\right)+M_{2}\left(\left|\begin{array}{ll}
P_{4 x} A_{x} & B_{x}^{\prime} \\
P_{1 x} A_{y} & B_{y}^{\prime}
\end{array}\right|+A_{x} B_{x}^{\prime}\left(P_{1 y}-P_{4 y}\right)\right)\right) \\
\left.+M_{3}\left(\left|\begin{array}{ll}
P_{4 x} B_{x} & A_{x}^{\prime} \\
P_{1 x} B_{y} & A_{y}^{\prime}
\end{array}\right|+B_{x} A_{x}^{\prime}\left(P_{1 y}-P_{4 y}\right)\right)+M_{4}\left(\left|\begin{array}{ll}
P_{4 x} B_{x} & A_{x}^{\prime} \\
P_{1 x} B_{y} & A_{y}^{\prime}
\end{array}\right|+B_{x} A_{x}^{\prime}\left(P_{1 y}-P_{4 y}\right)\right)\right] \triangleq\left|\begin{array}{ll}
\hat{\gamma}_{1} & \hat{\beta}_{1} \\
\hat{\gamma}_{2} & \hat{\beta}_{2}
\end{array}\right|, \\
\left|\begin{array}{ll}
\alpha_{1}^{\prime} & \beta_{1}^{\prime} \\
\alpha_{2}^{\prime} & \beta_{2}^{\prime}
\end{array}\right| \approx\left|\begin{array}{ll}
\alpha_{1} & \beta_{1} \\
\alpha_{2} & \beta_{2}
\end{array}\right|+\Delta_{x}\left[M_{1}\left|\begin{array}{ll}
A_{x} & A_{x}^{\prime} \\
A_{y} & A_{y}^{\prime}
\end{array}\right|+M_{2}\left|\begin{array}{ll}
A_{x} & B_{x}^{\prime} \\
A_{y} & B_{y}^{\prime}
\end{array}\right|+M_{3}\left|\begin{array}{ll}
B_{x} & B_{x}^{\prime} \\
B_{y} & B_{y}^{\prime}
\end{array}\right|+M_{4}\left|\begin{array}{ll}
B_{x} & A_{x}^{\prime} \\
B_{y} & A_{y}^{\prime}
\end{array}\right|\right] \triangleq\left|\begin{array}{ll}
\hat{\alpha}_{1} & \hat{\beta}_{1} \\
\hat{\alpha}_{2} & \hat{\beta}_{2}
\end{array}\right|,
\end{gathered}
$$

and 


$$
\begin{aligned}
\left|\begin{array}{ll}
\alpha_{1}^{\prime} & \gamma_{1}^{\prime} \\
\alpha_{2}^{\prime} & \gamma_{2}^{\prime}
\end{array}\right| \approx\left|\begin{array}{ll}
\alpha_{1} & \gamma_{1} \\
\alpha_{2} & \gamma_{2}
\end{array}\right| & +\Delta_{x}\left[M_{1}\left(\left|\begin{array}{cc}
P_{1 y} A_{x} & A_{x}^{\prime} \\
P_{4 y} A_{y} & A_{y}^{\prime}
\end{array}\right|+A_{y} A_{y}^{\prime}\left(P_{4 x}-P_{1 x}\right)\right)+M_{2}\left(\left|\begin{array}{cc}
P_{1 y} A_{x} & B_{x}^{\prime} \\
P_{4 y} A_{y} & B_{y}^{\prime}
\end{array}\right|+A_{y} B_{y}^{\prime}\left(P_{4 x}-P_{1 x}\right)\right)\right. \\
& \left.+M_{3}\left(\left|\begin{array}{ll}
P_{1 y} B_{x} & B_{x}^{\prime} \\
P_{4 y} B_{y} & B_{y}^{\prime}
\end{array}\right|+B_{y} B_{y}^{\prime}\left(P_{4 x}-P_{1 x}\right)\right)+M_{4}\left(\left|\begin{array}{ll}
P_{1 y} B_{x} & A_{x}^{\prime} \\
P_{4 y} B_{y} & A_{y}^{\prime}
\end{array}\right|+B_{y} A_{y}^{\prime}\left(P_{4 x}-P_{1 x}\right)\right)\right] \triangleq\left|\begin{array}{ll}
\hat{\alpha}_{1} & \hat{\gamma}_{1} \\
\hat{\alpha}_{2} & \hat{\gamma}_{2}
\end{array}\right|
\end{aligned}
$$

where $M_{1}, \ldots, M_{4}$ are some constants, $\left|\begin{array}{ll}\alpha_{1} & \beta_{1} \\ \alpha_{2} & \beta_{2}\end{array}\right|,\left|\begin{array}{ll}\gamma_{1} & \beta_{1} \\ \gamma_{2} & \beta_{2}\end{array}\right|$ and $\left|\begin{array}{ll}\alpha_{1} & \gamma_{1} \\ \alpha_{2} & \gamma_{2}\end{array}\right|$ are the corresponding noise-free terms. Thus, an approximation of the robot location $R$ becomes

$$
\hat{R}_{x}=\left|\begin{array}{ll}
\hat{\gamma}_{1} & \hat{\beta}_{1} \\
\hat{\gamma}_{2} & \hat{\beta}_{2}
\end{array}\right| /\left|\begin{array}{ll}
\hat{\alpha}_{1} & \hat{\beta}_{1} \\
\hat{\alpha}_{2} & \hat{\beta}_{2}
\end{array}\right|, \quad \hat{R}_{y}=\left|\begin{array}{ll}
\hat{\alpha}_{1} & \hat{\gamma}_{1} \\
\hat{\alpha}_{2} & \hat{\gamma}_{2}
\end{array}\right| /\left|\begin{array}{ll}
\hat{\alpha}_{1} & \hat{\beta}_{1} \\
\hat{\alpha}_{2} & \hat{\beta}_{2}
\end{array}\right| .
$$

Since (9) has the form of

$$
\hat{R}_{x}=\frac{E \Delta x+F}{I \Delta x+J}, \hat{R}_{y}=\frac{G \Delta x+H}{I \Delta x+J}
$$

for constants $E$ through $J$, we can obtain the following linear equation by eliminating $\Delta x{ }^{4}$

$$
\hat{R}_{y}=\frac{I H-G J}{I F-E J} \hat{R}_{x}+\frac{G F-E H}{I F-E J} .
$$

The above equation gives an approximated trajectory of the reconstructed robot locations, due to different image extraction errors $\Delta x$ added to $p_{4}$ in Fig. 2 (and Fig. 3).

In general, as will be demonstrated with simulation results in the next section, if the 2-D image error of a feature point is within a reasonably small range, it can be transformed approximately linearly into a planar region in the 3-D space of the reconstructed scene $e^{5}$. In particular, such a linear transformation of coordinate system will transform a circular region of image error into an elliptic one in the above planar region. Therefore, with only transformations of image error in two linearly independent directions, an approximate ellipse of reconstruction error can be obtained. Such error ellipses will be useful indicators for one to choose among point features in an image to establish the probabilistically most accurate planar location system using cross-ratios.

\section{Simulation Results}

We conduct a series of simulations for the error analysis of cross-ratio-based planar localization for a real robot with synthesized noises added to it, and some reference

\footnotetext{
${ }^{4}$ One can show that by applying Taylor series expansion to (8) and skipping the higher order terms, which gives a linear relationship between 2-D image extraction error and 3-D localization error, a linear equation identical to (11) can be obtained.

${ }^{5} \mathrm{~A}$ formal derivation of such a property is omitted for brevity.
} 
points, in an image. In these simulations, we consider the situation when extraction noises only affect a single image point. First, we investigate the characteristics of the localization error assuming 1-D noise along $x$-direction, as discussed in the previous section, as well as along other directions. Next, nominal boundary of an error ellipse due to two dimensional noises is computed to approximate that resulted from the circle of image inaccuracy. Finally, we give a cross-ratio-based localization scheme which adopts the proposed error analysis method to assist the selection of reference image points to optimize the reconstruction process.
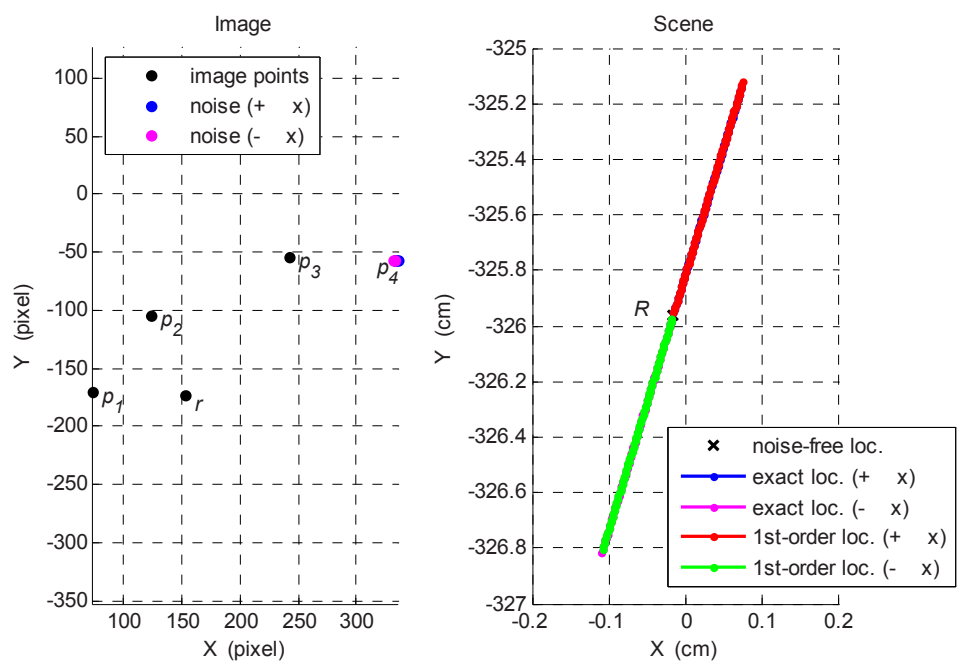

Fig. 4. (Left) Extracted feature points in an input image. $p_{1} \ldots p_{4}$ are identified as images of reference points, and $r$ is identified as robot image. Image extraction noises within a range of \pm 2 pixels along $x$-direction are added to $p_{4}$. (Right) Trajectory of reconstructed robot locations: blue and magenta points are obtained by (8) while red and green points are obtained by (12). The former are hardly visible since they are almost entirely covered by the latter. $R$ is the robot location in 3-D space resulted from noise-free extraction of image points.

Fig. 4 illustrates the trajectory of the reconstructed robot locations due to noises, within the range of \pm 2 pixels along $x$-direction, being added to $p_{4}{ }^{6}$. The locations obtained from linear equation (11) are represented in red and green colors, corresponding to deviations of $p_{4}$ into $+x$ and $-x$ directions, respectively. Points in blue and magenta colors represent similar results but computed with original rational equation (8). One can see that the latter, which are drawn first, are hardly visible since (11) gives a nearly perfect approximation of the former. In fact, similar results (which are omitted for brevity) can also be obtained for 1-D noises in arbitrary directions. In general, if the 2-D image errors are within a reasonable small range, the errors can also be transformed approximately linearly into the 3-D space of the reconstructed scene.

\footnotetext{
${ }^{6}$ It is assumed in the rest of the paper that two cross-ratios involved in the computation use $p_{1}$ and $p_{4}$ as origins, respectively.
} 

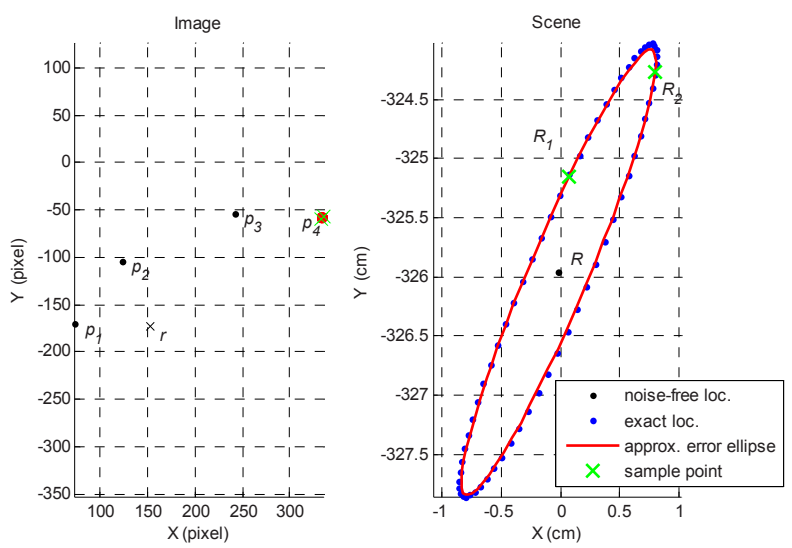

Fig. 5. (Left) Similar to that in Fig. 4 but with circularly distributed image extraction noises of \pm 2 pixels added to $p_{4}$. (Right) Trajectory of reconstructed robot locations: blue points are obtained by (8) while the approximate error ellipse is obtained using (12). The reconstructed locations of robot due to image errors $(\Delta x, \Delta y)=(2,0)$ and $(\Delta x, \Delta y)=(0,-2)$ on $p_{4}$ are at $R_{1}$ and $R_{2}$, respectively.
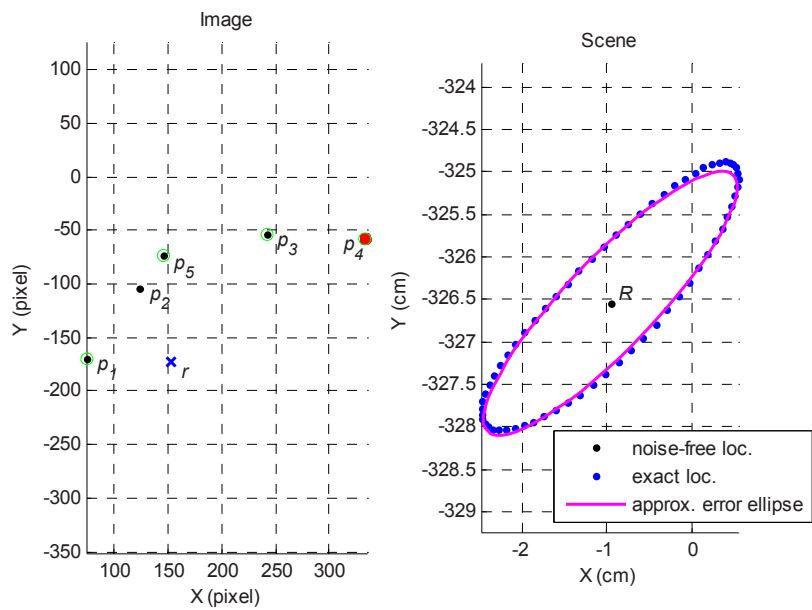

Fig. 6. Simulation results similar to that given in Fig. 5 but using reference point $p_{5}$ in place of $p_{2}$

Fig. 5 illustrates the trajectory of the reconstructed robot locations due to circularly distributed image extraction noises of 2 pixels added to $p_{4}$, as well as the error ellipse obtained from the linear transformation

$$
\left[\begin{array}{l}
\Delta R_{x} \\
\Delta R_{y}
\end{array}\right]=\left[\begin{array}{ll}
a & b \\
c & d
\end{array}\right]\left[\begin{array}{l}
\Delta x \\
\Delta y
\end{array}\right],
$$


where $\Delta R$ represents the corresponding location offsets derived from $\Delta x^{7}$. Specifically, we set $(\Delta x, \Delta y)=(2,0)$ and $(\Delta x, \Delta y)=(0,-2)$ (corresponding to reconstructed robot locations $R_{1}$ and $R_{2}$, respectively) and then derive the approximate error ellipse.

One can easily see that the above approximate error ellipse can be used to appropriately express the spatial characteristics of the localization error without the computation of a lot of reconstructed robot locations using the expensive high-order equation (8). For the application of a general cross-ratio-based localization algorithm, because a scene may have many image features (points) extracted, multiple choices of reference points, as well as the origins for the computation of cross-ratios, are possible. Fig. 6 shows simulation results similar to that given in Fig. 5 but using reference point $p_{5}$ in place of $p_{2}$. According to the results obtained with either (8) or (12), localization results in Fig. 6 give a similar worst case error, but with approximately twice the ellipse area, compared to that in Fig. 5.

The above results suggest that when there are multiple choices of reference points or cross-ratio origins, one can perform the proposed analysis to predict possible localization errors for each choice and select the optimal one accordingly. For each choice, one needs to ensure first that the noises are restricted to a reasonable range that (12) obtained using two noisy samples of the reference point of interest can appropriately describe the localization error. Subsequently, an optimal choice can be determined by comparing the direction of error, worst-case error, average error magnitude, or other metrics suggested by specific applications.

\section{Summary}

As a geometric invariant under projective transformations, cross-ratio is the basis of many recognition and reconstruction algorithms which are based on projective geometry. We propose an efficient way of approximately analyzing localization error for systems which use cross-ratio for planar localization, by establishing a linear relationship between localization error and small inaccuracy in measurements of image features due to 1-D and 2-D noises in the image space. Such an analysis will be useful for one to choose among point features, as well as cross-ratio origins, in stereo images to establish the probabilistically most accurate planar location system. The proposed approach is applicable whenever multiple choices of image features are available, which happens frequently in various computer vision applications, e.g. in robot navigation systems.

Acknowledgments. This work is partly supported by Ministry of Economic Affairs, Taiwan, under grant No. 95-EC-17-A-02-S1-032.

\section{References}

1. Kanatani, K.: Computational Cross-Ratio for Computer Vision. CVGIP 60, 371-381 (1994)

2. Mundy, J.L., Zisserman, A.: Geometric Invariance in Computer Vision. MIT Press, Cambridge, MA (1992)

${ }^{7}$ Eq. (12) can be used to derive the approximate error ellipse only if there is a linear relationship between image and reconstruction errors. Various ways of inspecting such a relationship exist, but is not discussed here for brevity. 
3. Lourakis, M.I.A., Halkidis, S.T., Orphanoudakis, S.C.: Matching Disparate Views of Planar Surfaces Using Projective Invariants. Image and Vision Computing 18, 673-683 (2000)

4. Carlsson, S.: Projectively Invariant Decomposition and Recognition of Planar Shapes. Int'l J. Computer Vision 17, 193-209 (1996)

5. Suk, T., Flusser, J.: Point-Based Projective Invariants. Pattern Recognition 33, 251-261 (2000)

6. Chuang, J.-H., Chiu, J.-M., Chen, Z.: Obtaining Base Edge Correspondence in Stereo Images via Quantitative Measures Along C-diagonals. Pattern Recognition Letters 18, 8795 (1997)

7. Chiu, J.-M., Chen, Z., Chuang, J.-H., Chia, T.-L.: Determination of Feature Correspondence in Stereo Images Using a Calibration Polygon. Pattern Recognition 30, 1387-1400 (1997)

8. Shashua, A., Navab, N.: Relative Affine Structure: Theory and Application to 3D Reconstruction From Perspective Views. IEEE Trans. Pattern Analysis and Machine Intelligence 18, 873-883 (1996)

9. Chuang, J.-H., Kao, J.-H., Chen, Y.-H.: Identity Verification by Relative 3-D Structure Using Multiple Facial Images. Pattern Recognition Letters 26, 1292-1303 (2005)

10. Nunziati, W., Sclaroff, S., Bimbo, A.D.: An Invariant Representation for Matching Trajectories Across Uncalibrated Video Streams. In: 4th Int'l Conf. Image and Video Retrieval, Singapore, pp. 318-327 (2005)

11. Rajashekahar, S.C., Namboodiri, V.P.: Image Retrieval Based on Projective Invariance. In: IEEE Int'l Conf. Image Processing, pp. 405-408. IEEE Computer Society Press, Los Alamitos (2004)

12. Tsonis, V.S., Chandrinos, K.V., Trahanias, P.E.: Landmark-Based Navigation Using Projective Invariants. In: IEEE/RSJ Int'l Conf. on Intelligent Robots and Systems, pp. 342-347 (1998)

13. Åström, K.: A Correspondence Problem in Laser Guided Navigation. In: Symposium on Image Analysis, Sweden, pp. 141-144 (1992)

14. Åström, K.: Automatic Mapmaking. In: 1st IFAC Int'l Workshop on Intelligent Autonomous Vehicles, Southampton, pp. 181-186 (1993)

15. Basri, R., Rivlin, E., Shimshoni, I.: Image-Based Robot Navigation Under the Perspective Model. In: Int'l Conf. Robotics and Automation, Michigan, pp. 2578-2583 (1999)

16. Guerrero, J.J., Sagüés, C.: Uncalibrated Vision Based on Lines for Robot Nnavigation. Mechatronics 11, 759-777 (2001)

17. Georgis, N., Petrou, M., Kittler, J.: Error Guided Design of a 3D Vision System. IEEE Trans. Pattern Analysis and Machine Intelligence 20, 366-379 (1998)

18. Huynh, D.Q.: The Cross-Ratio: A Revisit to its Probability Density Function. In: British Machine Vision Conf., pp. 262-271 (2000)

19. Åström, K., Morin, L.: Random Cross-Ratios. In: 9th Scandinavian Conf. on Image Analysis, pp. 1053-1060 (1995)

20. Maybank, S.J.: Stochastic Properties of the Cross-Ratio. Pattern Recognition Letters 17, 211-217 (1996)

21. Liu, J.-S., Chuang, J.-H.: A Geometry-Based Error Estimation of Cross-Ratios. Pattern Recognition 35, 155-167 (2002) 